\title{
Super-resolution Cryo-fluorescence Microscopy of High-pressure Frozen Thick Samples : A Screening Study of C. elegans
}

\author{
Kedar Narayan ${ }^{1}$, Irene Chang ${ }^{1}$, Mohammad Rahman ${ }^{2}$, Adam Harned ${ }^{1}$ and Orna Cohen-Fix ${ }^{2}$
}

${ }^{1}$ National Cancer Institute, NIH \& Frederick National Laboratory for Cancer Research, Frederick, Maryland, United States, ${ }^{2}$ National Institute of Diabetes and Digestive and Kidney Disease, NIH, Bethesda, Maryland, United States

Images of a specimen acquired by light and electron microscopy (LM, EM, respectively) have been combined and correlated in myriad ways [1], yet it is relatively difficult to precisely correlate 3D volume EM and LM datasets of thick samples. Dynamic biological processes are especially challenging as chemical fixation protocols introduce anisotropic warping of cellular structures [2], as well as an unavoidable time gap between physical addition and chemical reaction of the reagents. Newer in-resin fluorescence advances [3] solve spatial inaccuracies but provide little a priori temporal control and may not be universally applicable. Cryogenic fixation addresses these spatial and temporal issues. Indeed, commercially available cryo LM setups now allow samples, cryo-fixed on TEM grids, to be imaged by transmitted light and fluorescence techniques; these largely unaltered samples can then be imaged by cryo EM [4]. However, biological specimens thicker than a few micrometers have to be fixed by high-pressure freezing (HPF) in appropriate carriers in order to ensure proper cryopreservation of cellular structures [5].

HPF has been used successfully in a variety of settings for ultrastructural studies [5], but samples typically need to be further prepared after HPF in order to be rendered amenable to EM. The sequential steps involving freeze substitution, resin embedding and specimen trimming together require multiple days of working "blind" before images can be recorded. The difficulty of visualizing thick HPF derived samples by LM under cryogenic conditions has meant that researchers do not have a way to correlate or even quickly screen a sample post-HPF to check for spatiotemporal fidelity and freezing quality. We recently published 3D reconstructions of nuclear membrane architectures imaged by focused ion beam scanning electron microscopy (FIB-SEM) in the rapidly developing C. elegans embryo [6]. Membrane intermediates in these $\sim 50 \mu \mathrm{m} \times 30 \mu \mathrm{m}$ thick embryos are transient, requiring fixation by HPF to capture fleeting, yet crucial structures [7]. However, the inability to ascertain the presence of these structures until the very end of a laborious pipeline forced a slow and expensive "brute force" approach. Screening such thick samples for targeted intermediates would dramatically increase the efficiency and throughput of the imaging experiment.

Here, we have developed a procedure for imaging a thick specimen by cryo fluorescence microscopy, using $C$. elegans as a model system. Young adult worms measuring $\sim 0.8 \mathrm{~mm}$ in length and $60 \mu \mathrm{m}$ in diameter and containing embryos at various stages of development were collected in a cellulose capillary tube, affixed to a planchette, and high pressure frozen in an EM-ICE (Leica Microsystems, USA) in the presence of a variety of cryoprotectant combinations. These constructs were loaded into a planchette holder in a Linkam cryo stage (Linkam Scientific, United Kingdom) and imaged at low and high magnifications, either in normal or in Airyscan (super-resolution) mode on an upright Zeiss LSM 710 instrument (Carl Zeiss, Germany). We report that a subset of cryoprotectants tested show acceptable levels of low background fluorescence, low toxicity to the worm, and good structural preservation, allowing for targeted volume EM imaging. The ability to now screen samples by fluorescence for correct staging and quality of freezing could be of value for researchers, especially in situations where access to a volume EM instrument is limiting [8]. 


\section{References}

[1] P de Boer, JP Hoogenboom, BNG Giepmans, Nature Methods 12 (2015), p. 503.

[2] E Kellenberger et al., Journal of Microscopy 168 (1992), p. 181.

[3] CJ Peddie et al., Ultramicroscopy 143 (2014), p. 3.

[4] CM Hampton et al., Nature Protocols, 12 (2017), p. 150.

[5] D Studer, BM Humbel, M Chiquet, Histochemistry and Cell Biology 130 (2008), p. 877.

[6] M Rahman et al., Journal of Cell Biology 219 (2020), e201909137.

[7] MM Rahman et al., Molecular Biology of the Cell 26 (2015), p. 4718.

[8] This project has been funded in whole or in part with Federal funds from the National Cancer Institute, National Institutes of Health, under Contract No. 75N91019D00024. The content of this publication does not necessarily reflect the views or policies of the Department of Health and Human Services, nor does mention of trade names, commercial products, or organizations imply endorsement by the U.S. Government. 